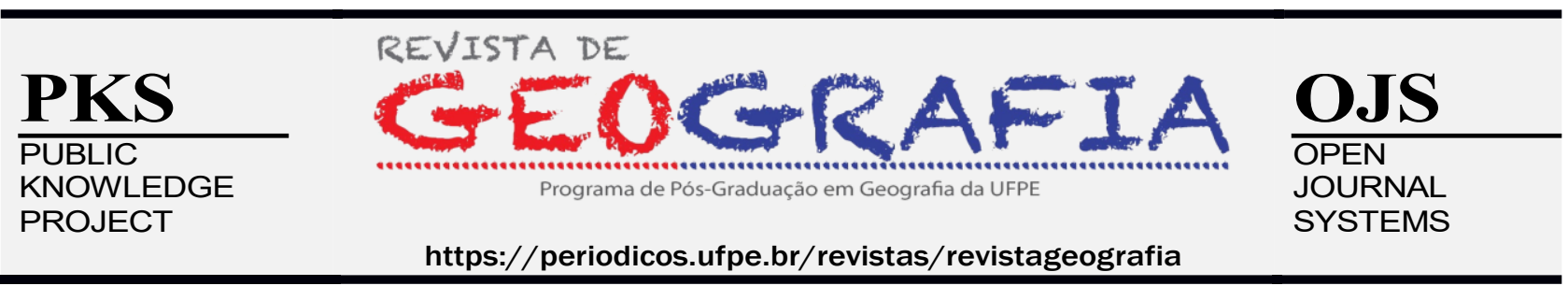

\title{
INTEGRAÇÃO DE SIG COM TÉCNICA AHP NA IDENTIFICAÇÃO DE LOCAIS COM VULNERABILIDADE A EROSÃO HÍDRICA DE UMA MICRO BACIA HIDROGRÁFICA EM CONTEXTO DE MATA ATLÂNTICA
}

\author{
Vinicius Emmel Martins ${ }^{1}$, Mireli Duarte Bergmann ${ }^{2}$, Marcio Augusto Reolon Schmidt ${ }^{3}$
}

${ }^{1}$ Universidade Federal do Paraná (UFPR),vinicius.emmel.m.92@gmail.com, Orcid: orcid.org/0000-0001-8532-4247
${ }^{2}$ Universidade Federal do Pampa (UNIPAMPA), mirelibergman@gmail.com, Orcid: orcid.org/0000-0001-9620-446X
${ }^{3}$ Universidade Federal de Uberlândia(UFU), marcio.schmidt@ufu.br, Orcid: orcid.org/0000-0003-2716-2360

Artigo recebido em 23/10/2020 e aceito em 19/07/2021

\section{RESUMO}

O presente trabalho tem como objetivo utilizar o método multicritério Analytic Hierachy Process (AHP), aplicado na busca de locais com vulnerabilidade à ocorrência de erosão hídrica, delimitando locais com risco de ocorrência de erosão considerando o aspecto laminar e linear. Os procedimentos metodológicos realizados no artigo foram primordialmente a comparação par a par e o cruzamento de cinco critérios de análises considerados decisivos para a determinação da vulnerabilidade: classe de solo, declividade, vigor da vegetação, uso do solo e intensidade de chuvas, bem como análise estatística das respostas dos especialistas, geração do autovetor de ponderação AHP e pôr fim a criação de um mapa utilizando o autovetor de ponderações AHP e o cruzamento dos cinco critérios, utilizando a Sistema de Informação Geográfica (SIG). Sete especialistas participaram da pesquisa. Áreas com maiores declividades e menor concentração de vegetação demonstraram tendências de serem locais com maior vulnerabilidade à ocorrência de erosão. Por outro lado, os locais com menor suscetibilidade à erosão, são justamente as regiões com uma declividade menos acentuada, encontramse geralmente próximas ao local de deságue do córrego Castelhano.

Palavras-chave: Analytic Hierarchy Process; Geographic Information System; Bacia Hidrográfica; Erosão.

\section{INTEGRATION OF SIG WITH AHP TECHNIQUE IN THE IDENTIFICATION OF PLACES WITH VULNERABILITY TO WATER EROSION OF A MICRO HYDROGRAPHIC BASIN IN THE ATLANTIC FOREST CONTEXT}

\section{ABSTRACT}

The present work aims to use the multicriteria Analytic Hierachy Process (AHP) method, applied in the search for places with vulnerability to the occurrence of water erosion, delimiting places with risk of erosion considering the laminar and linear aspect. The methodological procedures carried out in the article were primarily the pairwise comparison and the crossing of five analysis criteria considered decisive for determining 
vulnerability: soil class, slope, vegetation vigor, land use and rainfall intensity, as well as analysis statistics of the experts' answers, generation of the AHP weighting eigenvector and ending the creation of a map using the AHP weighting eigenvector and the crossing of the five criteria, using the Geographic Information System (GIS). Seven experts who participated in the research. Areas with higher declivity and less vegetation concentration showed a tendency to be places with greater vulnerability to the occurrence of erosion. On the other hand, the places with less susceptibility to erosion, are precisely the regions with a less pronounced slope, they were generally found close to the drainage point of the Castilian stream.

Keywords: Analytic Hierarchy Process; Geographic Information System; Hydrographic basin; Erosion.

\section{INTRODUÇÃO}

Os solos são recursos naturais importantes para o bem-estar do homem, compondo o ecossistema terrestre, integrado e influenciando na morfologia de bacias hidrográficas (PARKES et al. 2010). Os assoreamentos de rios também são frequentemente afetados por vários fatores que caracterizam o solo de sua região geográfica, entre eles a degradação do meio ambiente com impactos significativos, diminuindo as margens com vegetação densa de rios, córregos ou bacias, ocasionando erosões, afetando diretamente a integridade ambiental e a saúde humana (NOVARA et al. 2018; TREVISAN et al., 2018).

Segundo Fujihara (2002) os processos erosivos são caracterizados como desgaste, transporte e a deposição dos solos e das partículas de rochas, são classificadas conforme a sua intensidade, e divididos em dois grupos, o primeiro é o processo de erosão laminar, definido basicamente, como sendo a retirada da camada superficial de sedimentos do solo (FARINASSO et. al, 2006). E o segundo é a erosão linear decorrente da ação do escoamento hídrico superficial concentrado. A forma de erosão mais grave e com maiores impactos ambientais, apresentando divisão em três subgrupos: sulcos, ravinas e voçorocas (ORIENTI et al. 1996; FARINASSO et. al, 2006).

A prevenção de erosões acarreta em significativas melhorias na sustentabilidade e principalmente na capacidade dos recursos hídricos, podendo ajudar setores como a agricultura a realizar uma utilização sustentável e com rendimentos satisfatórios, proporcionando a mitigação de custos ambientais, sociais e financeiros (SOUZA, 2010; ZHOU e ZHANG, 2018). Trevisan et al., (2018), cita a finalidade de propor pesquisas sobre a utilização de ferramentas alternativas que auxiliem na manutenção da integridade ecológica e hidrológica, fomentando ações executadas precedentemente aos problemas de erosão ou semelhantes. Auxiliando na prevenção de acidentes ambientais e melhorando o planejamento e gestão de bacias hidrográficas.

Entre as pesquisas de geoprocessamento apresentadas na última década, destaca-se estudos com ênfase em técnicas inferidas a solos com vulnerabilidade a erosão. As mesmas possibilitam 
realizar os mapeamentos temáticos das feições ambientais, integrando e relacionando variáveis, desta forma possibilitando a identificação de locais propensos a processos erosivos (SILVA e MACHADO, 2014; TREVISAN et al., 2018). Como metodologias integradas a essas técnicas, o desenvolvimento do Sistema de Informação Geográfica (SIG) contribuiu para popularizar ferramentas aplicadas a estratégias globais, como por exemplo a previsão de problemas ambientais de curto e longo prazo (SCHMIDT e BARBOSA, 2016; CALDERANO et al., 2018; TEIXEIRA et al. 2019).

As técnicas de SIG possibilitam promover a integração de dados com origens diversificadas, o presente estudo se propõe a desenvolver uma metodologia que possibilita encontrar locais com potencialidades para ocorrências de erosão. O presente estudo propõe uma forma alternativa a abordagem deste problema ambiental, por meio da aplicação de uma metodologia que utiliza o processamento de informações geográficas, em conjunto com dados quantitativos obtidos a partir da opinião de especialistas no assunto, tratadas por meio do método multicritério Analytic Hierachy Process (AHP). Diante do exposto, o objetivo do presente trabalho é apresentar uma análise de conjunto de dados aptos que possibilitaram identificar locais com vulnerabilidade à ocorrência de erosão na microbacia do córrego Castelhano-RS.

\section{MATERIAIS E MÉTODOS}

O trabalho de investigação para identificar locais com suscetibilidade à erosão foi aplicado na região microbacia hidrográfica do córrego Castelhano, vegetação de Mata Atlântica, inserida no município denominado Venâncio Aires, Rio Grande do Sul. A bacia localiza-se nas coordenadas $29^{\circ}$ $36^{\prime} 00^{\prime} \mathrm{S} ; 52^{\circ} 10^{\prime} 00^{\prime} \mathrm{W}$, possui uma área total de $662,52 \mathrm{Km}^{2}$ ha Figura 2 - Mapa (1). O abastecimento de água neste município é realizado a partir das águas do córrego Castelhano. O arroio é um afluente do rio Taquari, pertencente à bacia hidrográfica Taquari Antas.

Para realização do trabalho, utilizou-se ferramentas de SIG na produção e análise dos mapas elaborados a partir do cruzamento de parâmetros estabelecidos por especialistas empregando o método AHP. As características morfométricas utilizadas para identificar locais com vulnerabilidade a erosão tanto laminar e linear foram: classe de solo, declividade, vigor da vegetação, uso do solo e intensidade de chuvas. O estudo de bacias hidrográficas e avaliação de riscos de erosão foi abordado metodologicamente como um problema multicritérios de tomada de decisão envolvendo múltiplos indicadores de avaliação, e sob este cenário, a técnica matemática AHP pode ser amplamente adaptada e utilizado para ponderar a importância dos indicadores (MARINS et al., 2009; BARBOSA 2016). As escolhas das respectivas classes para identificar locais com vulnerabilidade a erosão e que 
compõem o método AHP se basearam em estudos realizados por outros pesquisadores (NOVARA et al., 2018; ZHOU e ZHANG, 2018; SCHMIDT e BARBOSA, 2016; FALCI et al., 2015; SILVA e MACHADO, 2014; FEBRIAMANSYAH, 2006; ROSS, 1994). De modo sequencial as etapas principais do trabalho estão representadas em um fluxograma Figura 1.

\section{Figura 1. Fluxograma das etapas metodológicas adotadas no trabalho.}

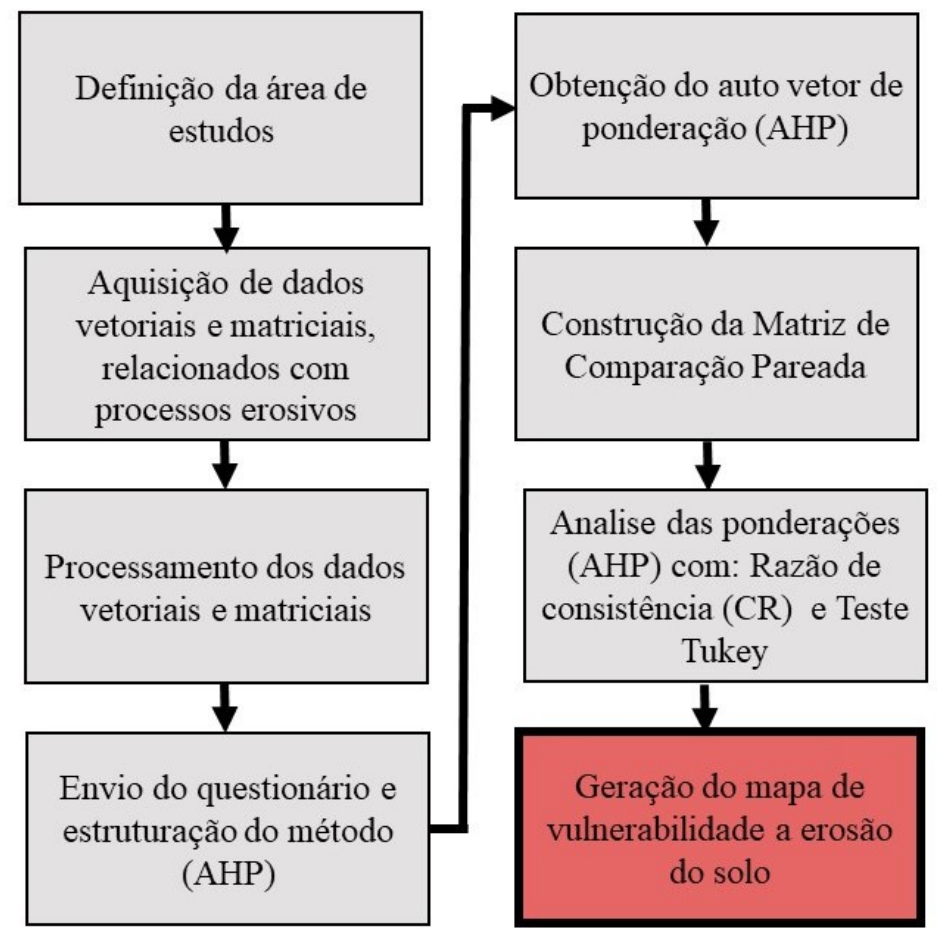

Fonte: Os Autores (2020)

A elaboração e aplicação da técnica AHP foi realizado por meio de questionário remoto, aplicado em um grupo de especialistas em geomorfologia, com formações em Engenharia Cartográfica e de Agrimensura, Engenharia Ambiental, Engenharia Agronomia e Geografia. O questionário foi formado por 10 questões, elaborado no Google Forms e enviado por e-mail, para os respectivos especialistas. As respostas possuíam uma escala de 1 a 9. Para obtenção de respostas com grau de coerência entre os especialistas, foi disponibilizado o peso dos critérios com o método AHP, com base no Quadro 1, cada pergunta continha um conjunto de mapas relativos Figura 2, posteriormente foi empregado a técnica Correlação Binária dos elementos, em cada nível hierárquico gerando uma matriz de decisão quadrada (SANTOS et al., 2016). 
Quadro 1: Intensidade da importância dos pesos.

\begin{tabular}{|c|l|l|}
\hline Pesos & \multicolumn{1}{|c|}{ Definição } & \multicolumn{1}{c|}{ Explicação } \\
\hline 1 & Mesma importância & As duas atividades contribuem igualmente para o objetivo. \\
\hline 3 & $\begin{array}{l}\text { Importância pequena de uma sobre a } \\
\text { outra }\end{array}$ & $\begin{array}{l}\text { A experiência e o julgamento favorecem levemente uma } \\
\text { atividade em relação à outra. }\end{array}$ \\
\hline 5 & $\begin{array}{l}\text { Importância grande ou essencial } \\
\text { A experiência e o julgamento favorecem fortemente uma } \\
\text { atividade em relação à outra. }\end{array}$ \\
\hline 9 & $\begin{array}{l}\text { Importância muito grande } \\
\text { oumonstrada }\end{array}$ & $\begin{array}{l}\text { Uma atividade é muito fortemente favorecida em relação à } \\
\text { outra; sua dominação de importância é demonstrada na prática. }\end{array}$ \\
\hline $2,4,6,8$ & $\begin{array}{l}\text { Valores intermediários entre os valores } \\
\text { adjacentes }\end{array}$ & $\begin{array}{l}\text { Ama designação razoável. } \\
\text { mais alto grau de certeza. }\end{array}$ \\
\hline
\end{tabular}

Fonte: Os Autores 2020.

A base de dados em shapefiles foi obtida no site do Instituto Brasileiro de Geografia e Estatística (IBGE) e da Agência Nacional de Águas (ANA). Os dados referentes aos Solos do Brasil escala 1:5.000.000 foram obtidos no site da Embrapa Solos. As imagens de satélite são oriundas do site do Instituto Nacional de Pesquisas Espaciais (INPE). 
Figura 2. Conjuntos de mapas utilizados

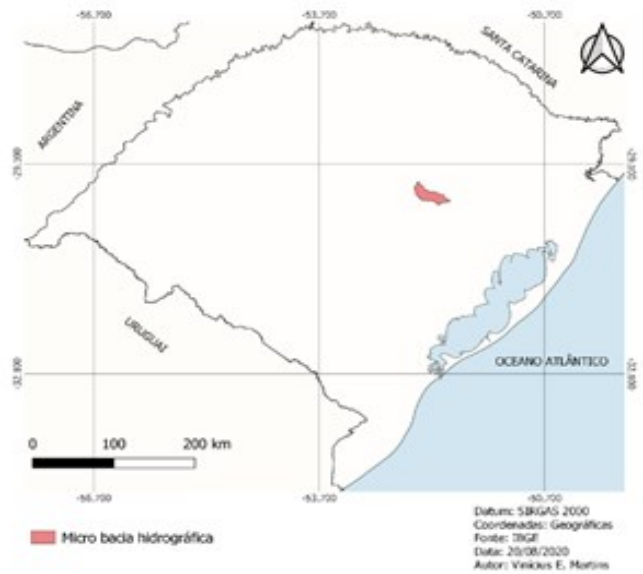

(1)

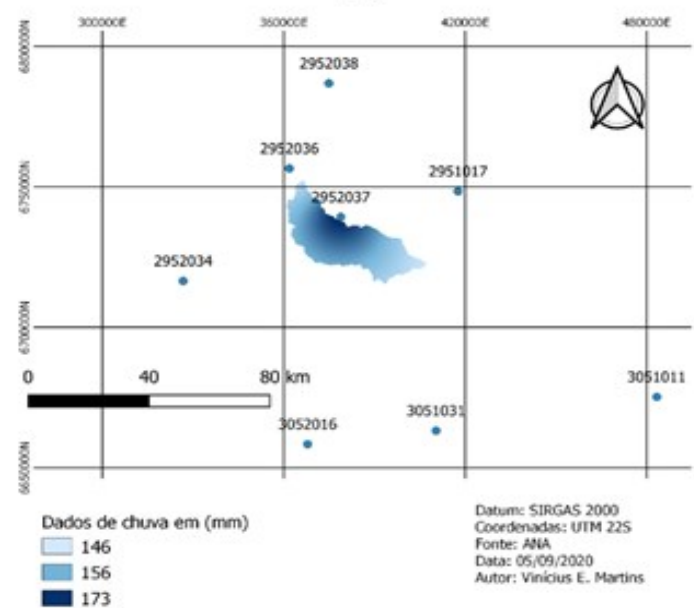

(3)

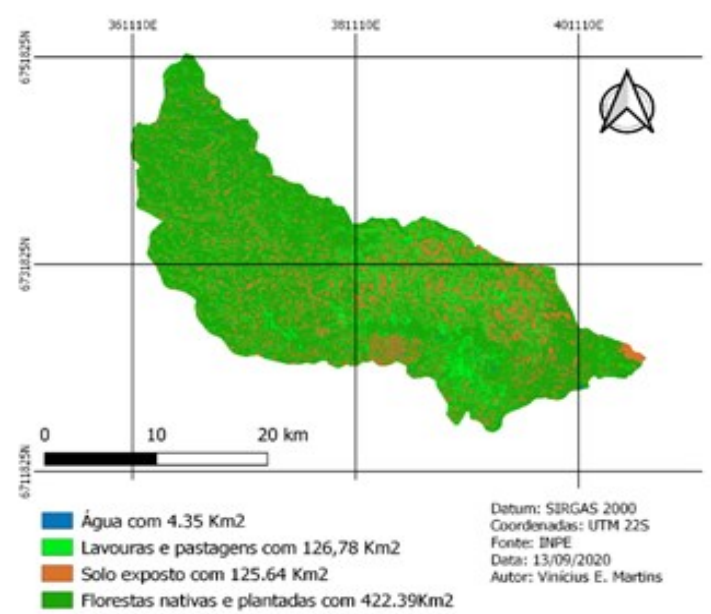

(5)

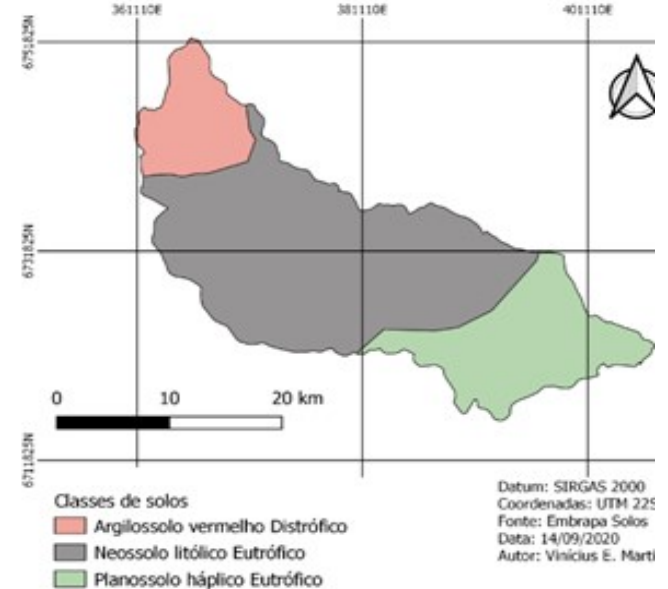

(2)

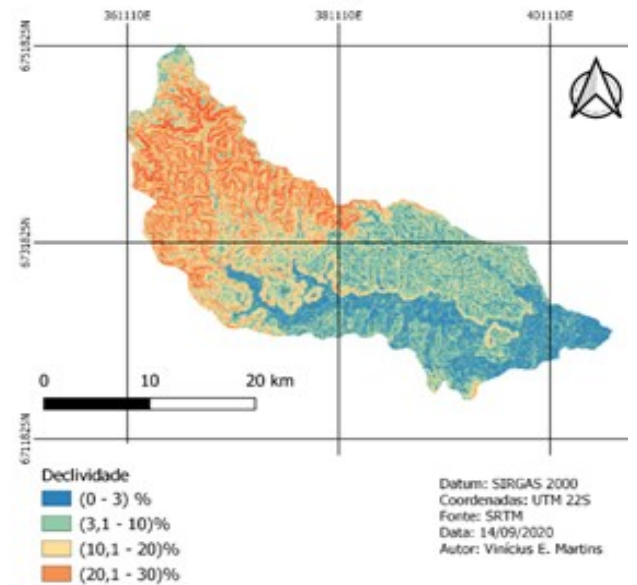

(4)

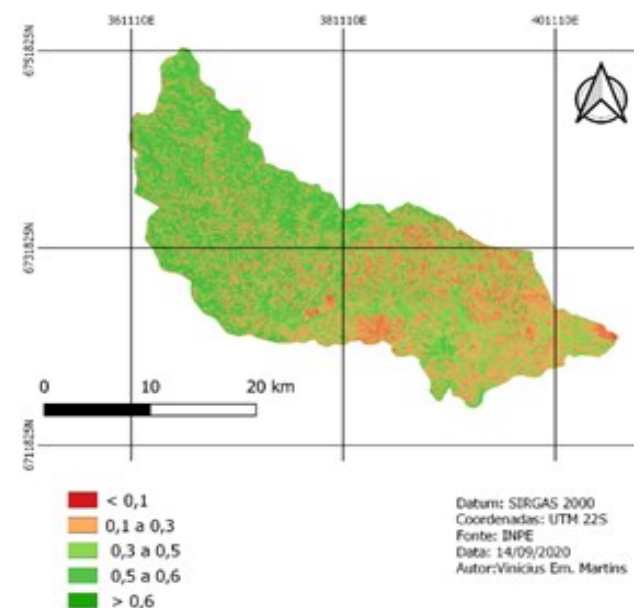

(6)

Fonte: Os Autores (2020). A composição da Figura 2 condiz ao mapa (1) correspondente a Localização da micro bacia hidrográfica do córrego Castelhano. O mapa (2) é referente à Classes de Solo da bacia do arroio castelhano. O mapa (3) trata do tema Estações pluviométricas interpoladas pelo método (IDW). A declividade do terreno corresponde ao mapa (4). O Uso e cobertura do solo mapa (5). Por fim, o mapa (6) coincide com o NDVI. 
A classificação das imagens supervisionada das imagens para gerar o mapa de "Uso do Solo" Figura 2 mapa (5) foram realizadas com a ferramenta Dzetsaka, adotando o modelo de Mistura Gaussiana que segundo Girard e Saracco (2016), apresenta ótimos resultados para determinar as classes oferecendo uma boa estrutura teórica para a seleção de modelos e para o cálculo de probabilidades de classificação, os modelos de Mistura Gaussiana são considerados ferramentas eficientes para classificações supervisionadas.

Foi utilizado as imagens do satélite CBERS-4 (Câmera Pancromática e Multiespectral (PAN10), utilizando as bandas 2, 3 e 4 com 10m composição colorida 342. Para medir a confiabilidade global da classificação supervisionada as imagens passaram por um procedimento denominado fusão utilizando a banda PAN $1 \mathrm{com} 5 \mathrm{~m}$ de resolução espacial, foi realizada uma avaliação através de uma imagem com melhor resolução espacial, que teve por finalidade comparar a classificação semiautomática ou supervisionada com a verdade terrestre.

O métodos de fusão das imagens empregado no trabalho foi o IHS (Intensity - I), matiz (Hue -H) e saturação (Saturation -S) no qual ocorre a transformação do sistema de cores RGB para o IHS, os componentes H e S que guardam a informação espectral das cores são preservados e o componente I é descartado e substituído pela imagem pancromática PAN 1 no caso do CBERS-4, para ser reamostrado os componentes $\mathrm{H}$ e $\mathrm{S}$ ficando com a resolução espacial da banda pancromática, sintetizando uma imagem colorida com resolução espacial da banda pancromática e com as cores do módulo multiespectral (MENESES e ALMEIDA 2007; PINHO et al. 2005).

O algoritmo utilizado no processo de fusão das imagens do satélite CBERS-4, foi o "i.pansharpen", do software Quantum SIG (QSIG) que usa uma imagem pancromática de melhor resolução para aumentar a resolução espacial das imagens multiespectrais de baixa resolução. Para analisar estatisticamente a confiabilidade dos dados foram utilizados a Matriz de Confusão e o coeficiente de concordância Kappa que fornece a concordância entre a classificação e os dados de referência. O algoritmo utilizado para gerar a matriz de confusão foi o "AcATaMa" e o cálculo do Kappa foi feito em uma planilha do Excel. Entre as recomendações para uso do Kappa, está a incorporação das informações dos pixels mal classificados, e não apenas dos bem classificados como a exatidão global (ROVEDDER, 2007; GOOVAERTS, 2002). O índice kappa obtido da classificação ficou acima de 74\%, o índice kappa avalia a qualidade de um mapeamento quando comparado com pontos de verdade terrestre (ESPÍRITO-SANTO e SHIMABUKURO 2005; GOMES e BIAS, 2018). 
Segundo Schmidt e Barbosa (2016), o método AHP foi desenvolvido por SATTY (1980; 1994). A comparação entre os fatores foi realizada com o propósito de definir a importância relativa de cada fator na opinião do especialista, expresso por meio de valores Quadro 1, comparando pares de dados. Os critérios de entrada do método são representados na forma de matriz, denominada matriz dominante Tabela 1, na qual as linhas e colunas recebem os valores relativizados de acordo com especialistas (SCHMIDT e BARBOSA, 2016).

Tabela 1: Matriz de comparação pareada ou matriz dominante

\begin{tabular}{c|ccccc}
\hline \multicolumn{1}{c}{ Fatores } & Classe de Solo & Declividade & $\begin{array}{c}\text { Vigor de } \\
\text { Vegetação }\end{array}$ & Uso do Solo & $\begin{array}{c}\text { Intensidade } \\
\text { das Chuvas }\end{array}$ \\
\hline Classe de Solo & 1 & $1 / \mathrm{P} 2$ & $1 / \mathrm{P} 3$ & $1 / \mathrm{P} 4$ & $1 / \mathrm{P} 5$ \\
Declividade & $\mathrm{P} 2$ & 1 & $1 / \mathrm{P} 2$ & $1 / \mathrm{P} 3$ & $1 / \mathrm{P} 4$ \\
Vigor de Vegetação & $\mathrm{P} 3$ & $\mathrm{P} 2$ & 1 & $1 / \mathrm{P} 2$ & $1 / \mathrm{P} 3$ \\
Uso do Solo & $\mathrm{P} 4$ & $\mathrm{P} 3$ & $\mathrm{P} 2$ & 1 & $1 / \mathrm{P} 2$ \\
Intensidade das Chuvas & $\mathrm{P} 5$ & $\mathrm{P} 4$ & $\mathrm{P} 3$ & $\mathrm{P} 2$ & 1 \\
\hline
\end{tabular}

Fonte: Adaptado de (SCHMIDT e BARBOSA, 2016; SAATY, 1990).

Após a obtenção dos valores pareados (P) entre os pares de fatores (Fn), divide-se cada elemento da coluna pela somatória da coluna a que pertence a Tabela 2. A média aritmética dos valores obtidos em cada linha, apresentadas na coluna à direita, representa os pesos normalizados (Pn). Estes pesos são o que Saaty (1994) chama de prioridade do parâmetro, ou seja, o peso inicial deste parâmetro na análise. O resultado desta etapa é um autovetor onde cada valor apresentado na coluna Pesos representa uma ponderação a ser atribuída na criação do mapa em SIG. Este autovetor é normalizado, ou seja, os pesos variam de 0 a 1 , sendo que a somatória de todos os pesos calculados foi 1 . 
Tabela 2: Caso genérico para determinação dos pesos

\begin{tabular}{|c|c|c|c|c|c|c|}
\hline Fatores & $\begin{array}{l}\text { Classe de } \\
\text { Solo }\end{array}$ & Declividade & $\begin{array}{l}\text { Vigor de } \\
\text { Vegetação }\end{array}$ & Uso do Solo & $\begin{array}{l}\text { Intensidade } \\
\text { das Chuvas }\end{array}$ & $\begin{array}{l}\text { Pesos n. } \\
\text { (Pn) }\end{array}$ \\
\hline $\begin{array}{l}\text { Classe de } \\
\text { Solo }\end{array}$ & $\begin{array}{c}\mathrm{F} 1 / \Sigma \\
\text { Coluna1 }\end{array}$ & $\begin{array}{c}\mathrm{F} 1 / \Sigma \\
\text { Coluna2 }\end{array}$ & $\begin{array}{c}\mathrm{F} 1 / \Sigma \\
\text { Coluna3 }\end{array}$ & $\begin{array}{c}\mathrm{F} 1 / \Sigma \\
\text { Coluna4 }\end{array}$ & $\begin{array}{c}\mathrm{F} 1 / \Sigma \\
\text { Coluna5 }\end{array}$ & $\sum$ Linhal/n \\
\hline Declividade & $\begin{array}{c}\mathrm{F} 2 / \Sigma \\
\text { Coluna1 }\end{array}$ & $\begin{array}{c}\mathrm{F} 2 / \Sigma \\
\text { Coluna2 }\end{array}$ & $\begin{array}{c}\mathrm{F} 2 / \Sigma \\
\text { Coluna3 }\end{array}$ & $\begin{array}{c}\mathrm{F} 2 / \Sigma \\
\text { Coluna4 }\end{array}$ & $\begin{array}{c}\mathrm{F} 2 / \Sigma \\
\text { Coluna5 }\end{array}$ & $\sum$ Linha $2 / \mathrm{n}$ \\
\hline $\begin{array}{c}\text { Vigor de } \\
\text { Vegetação }\end{array}$ & $\begin{array}{c}\mathrm{F} 3 / \Sigma \\
\text { Coluna } 1\end{array}$ & $\begin{array}{c}\mathrm{F} 3 / \Sigma \\
\text { Coluna2 }\end{array}$ & $\begin{array}{c}\mathrm{F} 3 / \Sigma \\
\text { Coluna3 }\end{array}$ & $\begin{array}{c}\mathrm{F} 3 / \Sigma \\
\text { Coluna4 }\end{array}$ & $\begin{array}{c}\mathrm{F} 3 / \Sigma \\
\text { Coluna5 }\end{array}$ & $\sum$ Linha3/n \\
\hline $\begin{array}{l}\text { Uso do } \\
\text { Solo }\end{array}$ & $\begin{array}{c}\mathrm{F} 4 / \Sigma \\
\text { Coluna1 }\end{array}$ & $\begin{array}{c}\mathrm{F} 4 / \Sigma \\
\text { Coluna2 }\end{array}$ & $\begin{array}{c}\mathrm{F} 4 / \Sigma \\
\text { Coluna3 }\end{array}$ & $\begin{array}{c}\mathrm{F} 4 / \Sigma \\
\text { Coluna4 }\end{array}$ & $\begin{array}{c}\mathrm{F} 4 / \Sigma \\
\text { Coluna5 }\end{array}$ & $\sum$ Linha $4 / \mathrm{n}$ \\
\hline $\begin{array}{l}\text { Intensidade } \\
\text { das Chuvas }\end{array}$ & $\begin{array}{c}\mathrm{F} 5 / \Sigma \\
\text { Coluna1 }\end{array}$ & $\begin{array}{c}\mathrm{F} 5 / \Sigma \\
\text { Coluna2 }\end{array}$ & $\begin{array}{c}\mathrm{F} 5 / \Sigma \\
\text { Coluna3 }\end{array}$ & $\begin{array}{c}\mathrm{F} 5 / \Sigma \\
\text { Coluna4 }\end{array}$ & $\begin{array}{c}\mathrm{F} 5 / \Sigma \\
\text { Coluna5 }\end{array}$ & $\sum$ Linha $5 / \mathrm{n}$ \\
\hline$\Sigma$ Colunas & 1 & 1 & 1 & 1 & 1 & 1 \\
\hline
\end{tabular}

Fonte: Adaptado de (SCHMIDT e BARBOSA, 2016; SAATY, 1990).

A consistência do método AHP foi estimada utilizando como referência no trabalho de Triantaphyllou e Mann (1995). O teste é considerado adequado se a relação correspondente de consistência (CR) é inferior a 10\%. O coeficiente IC é calculado utilizando a equação (1). Primeiro, o índice de consistência (IC) precisa ser estimado. Isto é feito através do somatório de colunas na matriz do julgamento, multiplicado pelo autovetor de ponderação AHP, onde $\lambda_{\text {max }}$ é uma aproximação do autovalor dos pesos obtidos.

$$
I C=\frac{\left(\lambda_{\max }-n\right)}{(n-1)}
$$

Dividindo-se o valor IC pelo índice aleatório IR Tabela 3 criado por Saaty (1994), apresentado na Tabela 1, para então obter um novo índice chamado de razão de consistência (CR) (SCHMIDT e BARBOSA, 2016; TRIANTAPHYLLOU e MANN, 1995). Os valores correspondentes a CR é recomendada que fique abaixo de 0,1 . 
Tabela 3: Valores de IR para matrizes de ordem n

\begin{tabular}{c|ccccccc}
\hline $\mathrm{n}$ & 1 & 2 & 3 & 4 & 5 & 6 & 7 \\
\hline $\mathrm{IC}$ & 0 & 0 & 0,58 & 0,90 & 1,12 & 1,24 & 1,32 \\
\hline
\end{tabular}

Fonte: Adaptado de Triantaphyllou e Mann (1995).

Após processamentos dos dados e elaboração de mapas temáticos, correspondente a classe de solo, declividade, vigor da vegetação, uso do solo e intensidade de chuvas. A elaboração e aplicação do método AHP para agrupar áreas que apresentaram, de acordo com as variáveis selecionadas, potencial de vulnerabilidade a erosão semelhante, através da análise de multicritério com o método de álgebra de mapas (SILVA e MACHADO, 2014).

Utilizando a ferramenta do software QSIG denominada “Calculadora Raster" que é considerada um algoritmo muito flexível e versátil que pode ser usado para muitas aplicações envolvendo dados raster (COELHO et al. 2013). Foi possível gerar o mapa com dados contínuos, correspondendo a vulnerabilidade à erosão. $\mathrm{O}$ formato raster contendo dados contínuos, passou por processamento de reclassificação, com base em intervalos de influência, que é um processo que realiza a distinção dos valores dos pixels (GUTIERREZ e BAUMANN, 2007). A camada do mapa de saída foi uma um mapa contendo dados discretos, com base na entrada de regras de reclassificação utilizando o algoritmo "r.reclass", disponível no software livre QSIG. Possibilitando ser calculado o valor das respectivas áreas utilizando o algoritmo "r.report", que permite ao usuário configurar uma série de parâmetros a serem aplicados ao mapa raster gerando um relatório final.

\section{RESULTADOS E DISCUSSÃO}

O método AHP possibilitou a adequação das características analisadas, de acordo com as necessidades e particularidades do problema estudado, conforme as circunstâncias do caso, permitindo a distinção dos componentes em classes de importância que permitiram uma visão geral das relações inerentes ao processo de erosão. Os resultados obtidos com a aplicação do método AHP fornecem uma abordagem conveniente para auxiliar na solução de problemas complexos, o peso de cada critério estudado no trabalho foi calculado e estes resultados serviram para apontar possíveis locais que possuem maior e menor vulnerabilidade à ocorrência de erosão, os pesos calculados estão apresentados na Tabela 4. 


\begin{tabular}{|c|c|}
\hline Componentes & Pesos (AHP) \\
\hline Tipos de solos & 0,04 \\
\hline Pluviometria & 0,08 \\
\hline Declividade & 0,14 \\
\hline Uso do solo & 0,27 \\
\hline NDVI & 0,47 \\
\hline TOTAL & 1 \\
\hline
\end{tabular}

Fonte: Os Autores 2020.

Os fatores ponderados pelos especialistas foram submetidos ao IR considerando a escala de 1,12 de valores IR para matrizes de ordem n Tabela 5, chega-se ao RC de 0,2. Porém este valor é maior do que 0,1 (10\%), o que não é recomendado segundo (TRIANTAPHYLLOU e MANN, 1995). O RC foi influenciado devido a técnica AHP apresentar algumas incertezas no processo de análise por não considerar principalmente o caráter subjetivo inerente às avaliações dos especialistas humanos nas ponderações. A maior dificuldade observada no uso desta metodologia consiste na atribuição de "pesos" às variáveis selecionadas. Pois, a definição e a atribuição de "pesos" é um fator determinante nos resultados finais, podendo conduzir a resultados variados, ou mesmo divergentes em função desta atribuição (CALDERANO et al., 2018). Esta discrepância é associada à multidisciplinaridade dos especialistas, onde cada especialista faz suas próprias observações e atribuições de pesos considerando seus saberes.

Sobre os fatores apresentados, ressalta-se o fator com maior importância, de acordo com os pesos atribuídos pelos especialistas, foi quantificada pelo NDVI, representando o fator vegetação. De acordo com os dados observados na Tabela 5, para 71,42\% dos especialistas entrevistados o fator "uso do solo" em comparação ao "vigor de vegetação", caracteriza-se como o agente de maior importância para que ocorra a erosão no solo. Este dado é corroborado por Lima et al, (2013) utilizando o NDVI para avaliar o dossel vegetativo relacionando o fator com a degradação em solos brasileiros pela erosão hídrica, presente na região analisada. A forma como mapas feitos a partir da aplicação da técnica NDVI apresenta os dados de vegetação permitem ao leitor facilidade no processo de identificação de áreas com cobertura vegetal pelo mapa (DEMARCHI et al, 2011; LIMA et al, 2013). Pode-se observar a análise associativa por meio de mapas entre a degradação vegetal e o 
processo erosão (BRAZ et al 2015; DECHEN et al. 2015). Contudo ressaltamos que os índices de vegetação não devem compor um parâmetro único para representar previsões sobre erosão do solo.

Apesar de percentualmente menor enfatizado pelos especialistas entrevistados, fatores como os apresentados nos fatores "uso de solo", "declividade" e "pluviometria", demonstram importância na análise em conjunto Tabela 3, interligando respostas Tabela 4. De acordo com Dechen et al. (2015) a ocorrência de processos erosivos é determinada, entre outros, por fatores como erosividade da chuva e uso do solo, mas também está diretamente relacionado às propriedades físicas, principalmente textura, estrutura, permeabilidade, densidade e espessura do solo. Braz et al (2015) demonstra que estas variáveis são componentes importantes. Sendo complementar o monitoramento contínuo da área para complementos atualizados de informações que favoreçam o planejamento e gestão ambiental de bacias hidrográficas (SILVA et al., 2009; BRAZ et al 2015).

A quantificação das áreas como maior e menor propensão em termos de suscetibilidade a erosão tanto laminar e linear são apresentados pela Tabela 5, na qual a área está na unidade de km2 e porcentagem de ocupação da sub bacia hidrográfica do córrego Castelhano, bem como a distinção em classes de vulnerabilidade.

Tabela 5. Área ocupada de acordo com grau de suscetibilidade a erosão.

\begin{tabular}{lll}
\hline Suscetibilidade & Área $\mathrm{km}^{2}$ & $\%$ \\
\hline Muito fraca & 213,82 & 32,27 \\
Fraca & 377,28 & 56,95 \\
Forte & 67,55 & 10,19 \\
Muito forte & 3,87 & 0,58 \\
\hline TOTAL & 662,52 & 100 \\
\hline
\end{tabular}

Fonte: Os Autores 2020.

De acordo com a Tabela 5 e Figura 3, as áreas de menor expressividade na bacia hidrográfica são as áreas com vulnerabilidade a ocorrência de erosão muito forte $(0,58 \%)$. O predomínio da classe de vulnerabilidade forte abrange aproximadamente (10,19\%) de ocupação da área total da microbacia hidrográfica. Locais com menor propensão a ocorrerem erosão corresponderam a vulnerabilidade 
fraca corresponde a $(56,95 \%)$ compondo a maior parte da microbacia hidrográfica, o índice de vulnerabilidade muito fraca foi de $(32,27 \%)$.

Figura 3: Mapa final de vulnerabilidade atribuído a erosão do solo.

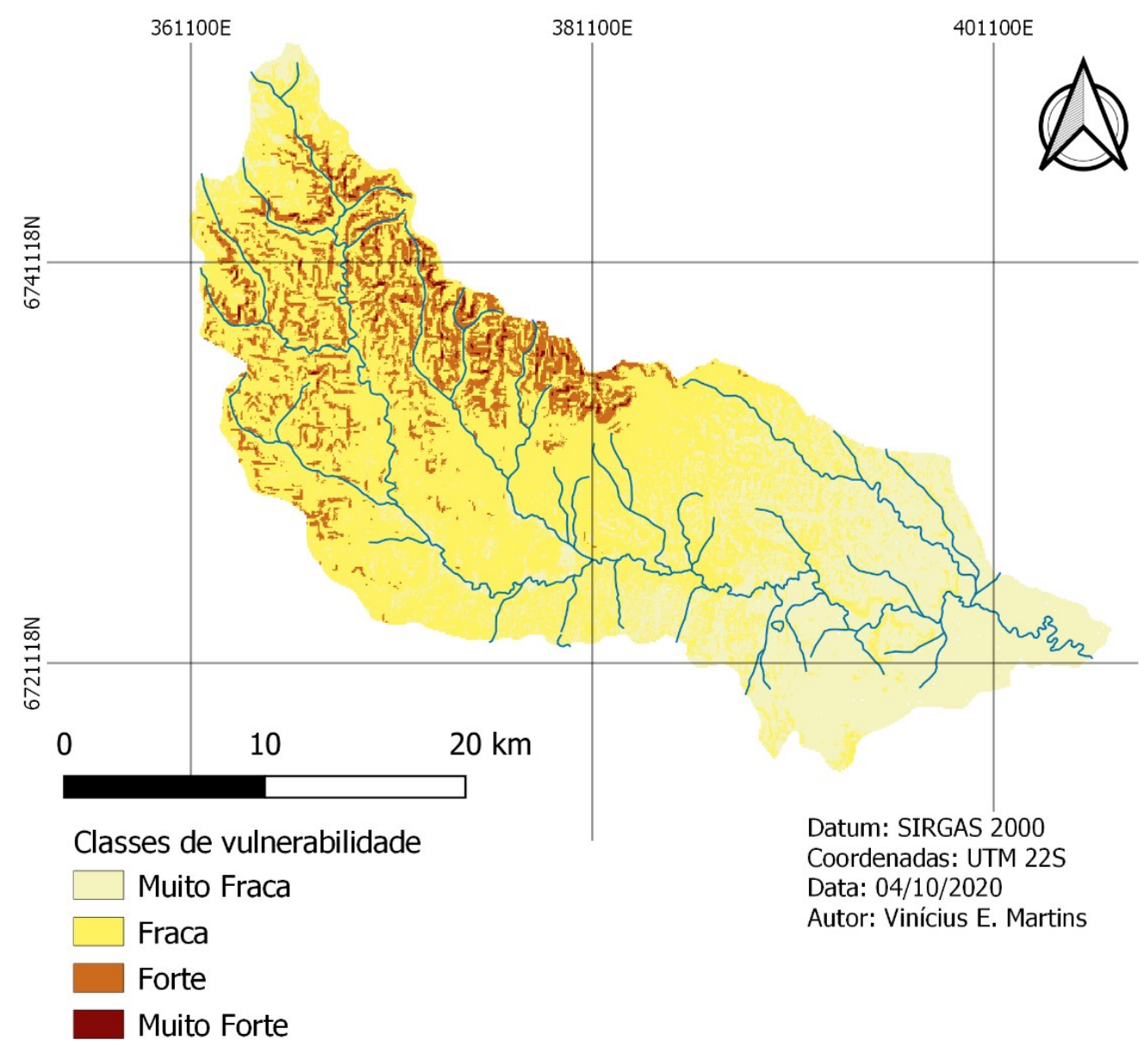

Fonte: Os Autores 2020.

O mapa Figura 3 contém a integração de todos os dados, possibilitando verificar quais locais são potencialmente vulneráveis a ocorrência de processos erosivos, os locais são classificados em quatro grupos, de acordo com o seu respectivo grau de vulnerabilidade, sendo as categorias "muito forte" e "forte" a ocorrer erosão, são locais que possuem um terreno com declividade acentuada. O elevado potencial de vulnerabilidade dos locais aos riscos ambientais, como movimentos de massa, erosão e escorregamentos acelerada dos solos, se deve principalmente às características topográficas do relevo montanhoso, associado aos índices de precipitação e às demais condicionantes geoambientais locais. 
A ocorrência da classe de solo denominada Neossolo Litólico Eutrófico, são solos com pequeno desenvolvimento pedogenético, geralmente caracterizados por baixa profundidade, possuindo camadas distinta herdadas dos materiais de origem, o que potencializa sua erosão, por meio de chuvas combinadas ao relevo acentuado. Em geral, os terrenos mais íngremes se preservam a cobertura florestal, atenuando a instabilidade da área, diminuindo o escoamento superficial e aumentando a capacidade de infiltração da água no solo, mas as interferências antrópicas na paisagem potencializam os riscos ambientais (MESQUITA et al., 2011; SILVA et al., 2015; CALDERANO et al., 2018). As áreas com menor suscetibilidade à erosão, são justamente os locais com uma declividade menos acentuada, encontram-se preferencialmente próximas do local de deságue do córrego Castelhano, ocorrendo inclusive o oposta da erosão que é a formação de depósitos sedimentares inconsolidados.

\section{CONSIDERAÇÕES FINAIS}

A erosão do solo é uma questão ambiental séria em todo o mundo, que afeta a vida e os recursos de milhões de pessoas, precisamos encontrar soluções eficientes como subsídio ao planejamento e programa de ações de conservação em bacias hidrográficas em todos os biomas, inclusive na Mata Atlântica. Ferramentas como SIG aliada à técnica AHP, demonstram boa eficiência na identificação de locais suscetíveis a erosão, com a hierarquização dos principais fatores que ocasionam a erosão hídrica. A integração dessas ferramentas sinaliza indicativos de que desenvolver pesquisas relevantes para a Geomorfologia tanto em âmbito de uma ciência global, ou mesmo regional como é o caso da proposta do presente estudo, fornecendo possibilidades para um eficaz planejamento ambiental de médio ou longo prazo.

A aplicação da técnica AHP deve ser utilizada principalmente na fase de planejamento, podendo apresentar incertezas atribuídas à realidade do campo e por isso não substitui verificações no local. Como trabalhos futuro é fundamental a realização de pesquisas relacionadas ao RC que ficou acima do valor recomendado por (TRIANTAPHYLLOU e MANN, 1995), avaliando qual o impacto real do RC, quando fica acima do valor recomendado, verificando a acurácia dos valores em relação a realidade e se existe alguma tolerância para se extrapolar os valores, dependendo do contexto de aplicação da técnica AHP, considerando que (TRIANTAPHYLLOU e MANN, 1995) estudaram o AHP e cunharam o índice RC com aplicações na Engenharia Industrial nos Estados Unidos. 
Portanto é imprescindível a realização de novos estudos levando em consideração o contexto que está sendo aplicado a técnica AHP e qual o impacto da multidisciplinaridade na formação dos especialistas, o que provoca abordagem e ponderações diferentes relacionadas a um problema específico condicionado a observações considerando seus saberes, sem muitas vezes estabelecer contato mais amplo com os saberes diferentes do seu.

\section{AGRADECIMENTOS}

À Coordenação de Aperfeiçoamento de Pessoal de Nível Superior (CAPES) pelo incentivo a pesquisa e apoio financeiro disposto no edital (16/2018-PPGCG e Resolução 32/17-CEPE).

\section{REFERÊNCIAS}

AGÊNCIA NACIONAL DE ÁGUAS (ANA). Disponível em: http://hidroweb.ana.gov.br (Acesso: 06 abril. 2020).

BRAZ, A. M.; DE ANDRADE Á. T.; GARCIA, P. H. M. Análise de índices de vegetação NDVI e SAVI e Índice de Área Folear (IAF) para a comparação da cobertura vegetal na bacia hidrográfica do córrego ribeirãozinho, município de Selvíria-MS. Revista Percurso, v.7, p.5-22, 2015.

CAlderano, F. B.; POlivanov, H.; CARVAlho J. W. D.; ChaGas, C. D. S.; CALDERANO, S. Avaliação da vulnerabilidade ambiental de regiões tropicais montanhosas com suporte de SIG. Revista de Geografia (Recife). V. 35, p. 269 - 288, 2018.

COELHO, A. L. N.; CORREA, W. S. C. Temperatura de Superfície Celsius do Sensor TIRS/Landsat8: metodologia e aplicações. Revista Geográfica Acadêmica, v.7, p.31-45, 2013.

DECHEN, S. C. F.; TELLES, T. T.; GUIMARÃES, M. F.; MARIA, I. C. Perdas e custos associados à erosão hídrica em função de taxas de cobertura do solo. Revista Brasileira de Ciência Bragantia, v.74, p.224-233, 2015.

DEMARCHI, J. C.; PIROLI, E. L.; ZIMBACK, C. R. L. Analise temporal do uso do solo e comparação entre os índices de vegetação NDVI e SAVI no município de Santa Cruz do Rio Pardo SP usando imagens LANDSAT-5. Raega Espaço Geográfico em Análise, v.21, p. 234-271, 2011.

EMPRESA BRASILEIRA DE PESQUISA AGROPECUÁRIA - EMBRAPA. Sistema brasileiro de classificação de solos. 2.ed. Brasília, 353p, 2006.

ESPÍRITO-SANTO, F. D. B.; e SHIMABUKURO, Y. E. Validação do mapeamento de uma área de floresta tropical com o uso de imagens de videografia aérea e dados de levantamento de campo. Revista Árvore, v.29, p.227-239, 2005.

FARINASSO, M., DE CARVALHO JÚNIOR, O. A., GUIMARÃES, R. F., GOMES, R. A. T., e RAMOS, V. M. Avaliação qualitativa do potencial de erosão laminar em grandes áreas por meio da 
EUPS equação universal de perdas de solos utilizando novas metodologias em SIG para os cálculos dos seus fatores na região do Alto Parnaíba PI-MA. Revista Brasileira de Geomorfologia, v.7, p.73$85,2006$.

FEBRIAMANSYAH, R. The use of AHP (the analytic hierarchy process) method for irrigation water allocation in a small river basin (case study in Tampo river basin in West Sumatra, Indonesia). In: the 11th Conference of the International Association for the Study of Common Property. 2006.

FUJIHARA, A. K. Predição de erosão e capacidade de uso do solo numa micro bacia do oeste paulista com suporte de geoprocessamento. Dissertação de Mestrado. Escola Superior de Agricultura Luís de Queiroz-Universidade de São Paulo, 118p, 2002.

GIRARD, S.; SARACCO, J. Supervised and unsupervised classification using mixture models. EAS Publications Series, v.77, p. 69-90, 2016.

GOMES, R. D. C., E BIAS, E. S. Integração do método AHP e sig como instrumento de análise do nível de conservação ambiental em bacias hidrográficas. Geociências (São Paulo), v.37, p.167-182, 2018.

GOOVAERTS, P. Geostatistical incorporation of spatial coordinates into supervised classification of hyperspectral data. Journal of Geographical Systems, v.4, p.99-111, 2002.

GUTIERREZ, A. G., E BAUMANN, P. Modeling fundamental geo-raster operations with array algebra. In Seventh IEEE International Conference on Data Mining Workshops (ICDMW 2007), p. 607-612, 2007.

INSTITUTO BRASILEIRO DE GEOGRAFIA E ESTATÍSTICA (IBGE). Manual técnico de pedologia. Coordenação de Recursos Naturais e Estudos Ambientais. 2ed. Rio de Janeiro: IBGE, 2007. 316 p. Disponível em: <http://biblioteca.ibge.gov.br/visualizacao/livros/liv37318.pdf>. (Acesso: 5 Junho de 2020).

LIMA, G. C.; SILVA, M. L. N.; CURI, N.; SILVA, M. A. D.; OLIVEIRA, A. H.; AVANZI, J. C.; UMMUS, M. E. Avaliação da cobertura vegetal pelo índice de vegetação por diferença normalizada (IVDN). Revista Ambiente \& Água, v.8, p.204-214. (2013).

MARINS, C. S.; SOUZA, D. O.; BARROS, M. S. O uso do método de análise hierárquica (AHP) na tomada de decisões gerenciais-um estudo de caso. In: XLI SBPO 2009 - Pesquisa Operacional na Gestão do Conhecimento, p.1778-1788, 2009.

MENESES, P. R.; ALMEIDA, T. Introdução ao processamento de imagens de sensoriamento remoto. Brasília: UNB/CNPq, 276p, 2007.

MESQUITA, C.; DE ASSIS, A. Q. S.; DE SOUZA, R. M. Vulnerabilidade natural à perda de solos da bacia hidrográfica do Rio Sagrado-Morretes/PR. Revista de Geografia (Recife), v.27, p.251-264, 2011. 
NOVARA, A.; PISCIOTTA, A.; MINACAPILLI, M.; MALTESE, A.; CAPODICI, F.; CERDÀ, A.; GRISTINA, L. The impact of soil erosion on soil fertility and vine vigor. A multidisciplinary approach based on field, laboratory and remote sensing approaches. Science of the Total Environment, v.622, p.474-480, 2018.

ORIENTI, I., AIEDEH, K.; GIANASI, E.; BERTASI, V.; ZECCHI, V. Indomethacin loaded chitosan microspheres. Correlation between the erosion process and release kinetics. Journal of microencapsulation, v.13, 463-472, 1996.

PARKES, M. W.; MORRISON, K. E.; BUNCH, M. J.; HALLSTRÖM, L. K.; NEUDOERFFER, R. C.; VENEMA, H. D.; WALTNER-TOEWS, D. Towards integrated governance for water, health and social-ecological systems: The watershed governance prism. Global Environmental Change, v.20, p.693-704, 2010.

ROSS, J. L. S. Análise Empírica da Fragilidade dos Ambientes Naturais e Antropizados. Revista do Departamento de Geografia, v.8, p.63-74, 1994.

ROVEDDER, J. Validação da classificação orientada a objetos em imagens de satélite Ikonos II e elaboração de indicadores ambientais georreferenciados no município de Torres, Planície Costeira do Rio Grande do Sul. Universidade Federal do Rio Grande do Sul. Centro Estadual de Pesquisas em Sensoriamento Remoto e Meteorologia. Programa de Pós-Graduação em Sensoriamento Remoto, Tese de doutorado, 83p, 2007.

SANTOS, M.; SOUZA, H. H.; DIAS, F. C.; REIS, M. F.; SANTOS, F. M. C. Aplicação do Método AHP na formação de um portfólio de projetos: um estudo de caso na área de TI de uma empresa sem fins lucrativos no Estado do Rio de Janeiro. Revista Produção, v.3, p.15-27, 2016.

SAATY, T. L. "How to make a decision: the hierachy process". European Journal of Operational Research. Elsevier Science Publisher, North Holland. v.48, p.9-26, 1990.

SAATY, T. L. Highlights and critical points in the theory and application of the Analytic Hierarchy Process. European Journal of Operational Research. v.74 p.426-447, 1994.

SCHMIDT, M. A. R.; BARBOSA, G. R. Uso de redes neurais artificiais na ponderação inicial da técnica AHP em análises de vulnerabilidade de bacias hidrográficas. Boletim de Ciências Geodésicas, v.22, p.511-525, 2016.

SILVA A. L. N. P.; DE SOUZA, C. A.; GALBIATI, C.; DOS SANTOS L. G. R. Caracterização e análise estatística da erosão marginal na sub-bacia hidrográfica do córrego das Pitas-Mato Grosso. Revista de Geografia (UFPE), v.32, 2015.

SILVA, V. C. B.; MACHADO, P. S. SIG na análise ambiental: susceptibilidade erosiva da bacia hidrográfica do córrego Mutuca, Nova Lima-Minas Gerais. Revista de Geografia (UFPE), v.31, p.66-87, 2014. 
SOUZA, J. C. Evaluation of the susceptibility and potential the laminar erosion of the watershad of stream Sozinha (GO). Dissertação (Mestrado em Ciências Humanas) - Universidade Federal de Goiás, Goiânia, 101p, 2010.

TEIXEIRA, M. B.; CAMARGO, P. L. T.; JUNIOR, P. P. M. Uso das Imagens de Sensores Remotos para Análise Crítica da Degradação do Cerrado no Alto Médio São Francisco-Minas Gerais. Anuário do Instituto de Geociências, v.41, p.245-254, 2019.

TREVISAN, D. P.; MOSCHINI, L. E; TREVISAN, B. P. Avaliação da vulnerabilidade dos solos à erosão no munícipio de São Carlos-SP. Revista de Geografia (Recife), v. 35, n. 2, p. 354-370, 2018.

TRIANTAPHYLLOU, E.; MANN, S.H. Using the analytic hierarchy process for decision making in engineering applications: some challenges. International Journal of Industrial Engineering: Applications and Practice, P.35-44, 1995.

ZHOU, J. L.; XU, Q. Q.; ZHANG, X. Y. Water Resources and Sustainability Assessment Based on Group AHP-PCA Method: A Case Study in the Jinsha River Basin. Water, v.12, p.0-23, 2018. 